\title{
Violencia familiar y su impacto sobre el niño
}

\author{
Fresia Ulloa Ch.
}

\section{Effects of family violence in children}

\begin{abstract}
Fomily violence is foirly prevalent problem in our society. There are often serious sequelae for obused children and for children who wilness different forms of domestic violence (physical, emotional or sexual|. Fomily viclence affects children nol only incirectly as witress but also as target or victim of agression and in several aspects. Niegative effects of these abnormal relationships cre potentially longterm and they moy even be ronstered to the following generations. A better understanding of these issues will enhonce comprehensiveness of protessional support cnd provide a betler outcorre for children and their tamilies.
\end{abstract}

\{Koy words: child abuse, family violence, purishment.\}

Aunque las investigaciones en esta área son relativamente recientes, la violencia familiar ha sido reconocida desde la antiguedad. En 1985 se encontro, en momias de hace 2000 a 3000 años, una incisncia mucho mayor de fracturas entre las mujeres, cuyo análisis concluyo que habrían resultado de "violencia en tiempos de paz". El maltrato a mujeres y niños, las formas mas frecuentes de violencia doméstica, fue amparado por la ley hasta comienzos del siglo $\mathrm{XX}^{1-5}$. En 1871 , una niña pequeña fue severamente golpeada por sus padres adoptivos en Nueva York: Los vecinos informaron de las lesiones que sufrí a la "Sociedad para la Prevención de la Crueldad en Animales", que en esa época era la única autoridad legal disponible. Poco tiempo después se creó la "Sociedad para la Prevención de la Crueldad en Niños"; sin embargo, sólo en 1961 Henry Kempe describio el "síndrome del niño golpeado", contribuyendo al reconocimiento del abuso infantil como un problema importante y frecuente ${ }^{2,6}$.

Si bien parte del abuso de niños es hecho por extraños, $85 \%$ de los casos se deben a personas que el niño conoce, por lo general el padre o la madre. Niños de todas las edades y condiciones socioeconómicas son víctimas de abuso físico,

1. Unidad de salud mental infantil y juvenil, Servicio de Salud Metropolitano Norte. psíquico o sexual cometido por los padres verdaderos, sustitutos, abuelos u otros miembros de la familia en sus propios hogares $2,3,7,8$, La violencia ocurre en todas las clases sociales y afecta a todos los miembros de la familia, especialmente a los niños, que suelen ser testigos y, en alta proporción, también víctimas de ésta ${ }^{3,6}$, a pesar de lo cual las consultas por este problema suelen ser escasas $1,2,5,8 \cdot 10$.

\section{Definición de violencia intrafamiliar $y$ maltrato infantil}

Aún no hay consenso en una definición de violencia intrafamiliar o doméstica. Esta alude a todas las formas de abuso que ticnen lugar en las relaciones entre los miembros de una familia ${ }^{11}$. La violencia intrafamiliar es toda acción u omisión cometida por un miembro de una familia, que menoscaba la vida, integridad física, psicológica o la libertad de otro miembro de ella y causa daño al desarrollo de la personalidad del agredidon.

Para poder calificar una situación familiar como caso de violencia doméstica, el abuso debe ser de cierta duracion, permanente o periodico, de modo que no se incluyen en ella el maltrato infrecuente o esporádico, que constituye la excepción -más que la regla- en las relaciones familiares ${ }^{11,12}$. La violencia familiar, en el sentido amplio de las distintas fommas de rela- 
ción abusiva que caracterizan de modo permanente o cíclico al vínculo intrafamiliar, puede comprometer a cualquier miembro de la familia, independientemente del sexo o edad, como agente o víctima de la relación abusiva; sin embargo, las mujeres y los niños son las víctimas más comunes ${ }^{11}$.

Grillo entiende por maltrato infantil toda acción u omisión por parte de un sujeto individual o colectivo, que violente el derecho de cualquier persona menor de edad a crecer y desarrollarse, independientemente de que tal acción u omisión sea producto de negligencia, ignorancia o incapacidad, ocurra en la intimidad del domicilio familiar o en un ámbito más amplio de la socie$\mathrm{dad}^{13}$. Estas acciones $\mathrm{u}$ omisiones interfieren en el crecimiento y desartollo físico, psíquico o sexual de tal menor ${ }^{11,13}$.

Se entiende por maltrato físico en el niño toda agresión física no accidental por parte de los padres o cuidadores, que como producto de castigo -único o repetido- le provoque daño físico o enfermedad de magnitud y características variables con consecuencias observables y medibles. El maltrato emocional es el hostigamiento verbal habitual de un niño a través de insultos, críticas, descrédito y ridiculización, el constante bloqueo de sus iniciativas, la indiferencia o rechazo explícito o implícito. El abandono y negligencia en los cuidados es la falta de protección y atención físicas mínimas del niño por parte de sus custodios, así como la falta de respuesta a sus necesidades evolutivas, por falta de estimulación cognitiva, que no se deba a carencia extrema de recursos sociales o económi$\cos$. Se considera abuso sexual cualquier forma de contacto o actividad de este tipo entre un niño y un adulto, ocasional o sostenida en el tiempo, incluyendo la explotación en comercio sexual ${ }^{1-3,5-9,13-16}$. Los niños testigos de violencia son aquellos que presencian situaciones crónicas de ésta entre sus padres u otros miembros de su familiall, 15.

\section{Hipótesis explicativas del maltrato infantil}

Hay diversas explicaciones para el fenómeno del inaltrato infantil, entre ellas destacan como relevantes las que se mencionan a continuación.

Factores culturales: la familia se encuentra inmersa en un ambiente social y cultural espe- cífico y comparte con este sistema un conjunto de valores y pautas de conducta que regulan la convivencia. Dentro de éstos está la del castigo físico como un método apropiado para educar y corregir a los niños. Esta creencia, por otra parte, va aparejada a desconocimiento o desvalorización de otros métodos educativos como la caricia, el reconocimiento, el halago, considerados como formas de niala crianza $1,3,5-7,11,16-20$. Entre estudiantes secundarios y universitarios ciertas conductas evidentemente violentas, como el zamarreo, no son consideradas como tales por ellos ${ }^{5}$. En una muestra de 527 adultos chilenos, $4,7 \%$ declaraba que el castigo era inútil, pero $75,5 \%$ lo utilizaba ${ }^{7}$. Es más, hay desacuerdo entre los profesionales, y también entre los padres, acerca de qué constituye disciplina y qué abuso físico; como asimismo entre lo que es cuidado adecuado y negligencia ${ }^{5}$. Algunos modelos educativos preconizan la violencia como medio de aprendizaje y ciertas estructuras sociales toleran o $\rightarrow$ al menos- no prohíben el empleo de la violencia en las relaciones con los nin̆os, estimando la agresividad individual como un medio para obtener buen éxito ${ }^{18}$. Puesto que el niño que vive la violencia aprende el modo agresivo de interacción y resolución de conflictos y llega a considerar natural y adecuado el uso de ésta, el modelo de aprendizaje social utilizado explica la transmisión intergeneracional de la violencia como conducta aprendj$\mathrm{da}^{2}, 6.12,17-19,21.22$.

Trastornos psiquiátricos: determinadas alteraciones en la salud mental de los padres pudiesen ser factores causales importantes de maltrato infantil. Los trastornos que parecen más frecuentemente relacionados con conducta abusiva son el alcoholismo, la drogadicción, neurosis, psicosis y retardo mental ${ }^{1-3,6,9,12,17,23}$.

Cuando hay problemas en el establecimiento del vínculo o desapego, no se desartollan vínculos afectivos profundos, estables o duraderos entre los padres y sus hijos. En estos casos, los primeros no son capaces de percibir ni satisfacer las necesidades y demandas de los niños ${ }^{2.6}$ 12. 17. En otras oportunidades los padres pueden tener expectativas que no consideren las capacidades reales de desempeño de los niños, lo que acumula frustraciones en los progenitores y aumenta las probabilidades de castigo físico. En esta misma situación se encuentran los niños con características que se diferencian de las del 
resto de la familia o de las esperadas por los padres $6,9,17,18$. Puede también haber dificultades propias de los sujetos agredidos, entre ellas las discapacidades o desventajas físicas, mentales a conductuales del niño, pueden provocar rechazo en lugar de conductas protectoras, lo que disminuye las posibilidades de estimulación $y$, por ende, de desarrollo del afectado ${ }^{17}$.

La idea que el hijo es propiedad de sus progenitores existe en nuestro sistema de valores $e$ implica que éstos tienen el derecho a actuar y utilizar el castigo como medida disciplinaria ante el niño, cada vez que lo estiman conveniente, situación en la que, por ser un "asunto privado", nadie puede ni debe intervenir ${ }^{11,}$ 17, 19 .

Circunstancias agobiantes: ante determinados eventos traumáticos persistentes puede ser excedida la capacidad de tolerancia y adaptación de una persona, la cual reacciona eventualmente en forma agresiva con otros miembros de su familia ${ }^{1,4}, 12,17.13,20.23$.

Factores socioeconómicos: la insuficiencia de recursos sociales y económicos puede crear tensión y apremio dentro de la familia, pudiendo los niños llegar a ser considerados como una carga $a$ motivo de incomodidad en tales circunstancias $^{12,} 18$.

La familia es considerada como uno de los pilares fundamentales de nuestra sociedad, es donde encontramos afecto y apoyo frente a la adversidad, donde se nos entrega el soporte afectivo necesario para desarrollar plenamente nuestras capacidades, donde nos desarrollamos como personas y donde aprendemos los valores, conductas y roles sociales. La violencia doméstica es un fenómeno complejo. Es difícil enfrentar y reconocer que al interior de la familia, en donde existe, por lo general, afecto entre sus miembros, se dé este tipo de situaciones ${ }^{11,13}$. Diversos autores señalan que los mismos factores que constituyen fortalezas en las familias sanas, pueden predisponer a la violencia en las familias que viven violencia. Estos factores son el grado de compromiso afectivo, la cantidad de tiempo que se pasa juntos, las oportunidades para compartir con otros miembros de la familia, el amplio rango de actividades e intereses, los derechos implícitos a influir la conducta del otro, desigualdad que resulta de la organización jerárquica de la familia, privacidad del sistema, alto nivel de estrés y el conocimiento extenso que cada miembro del sistema tiene del otro ${ }^{12.13 .21}$. En relación a esto, se ha visto que las madres son las que con mayor frecuencia maltratan a sus hijos; se cree que esto sería resultado no de una propensión de las mujeres hacia la violencia, sino más bien a la cantidad de tiempo que deben compartir con el niño $0^{5.7}$.

Grillo plantea, en base al modelo de Finkelhor, que existen cuatro precondiciones al maltrato infantil. El abuso de nir̃os y niñas sería el resultado de un proceso. La primera precondicion es la presencia de un abusador potencial, con una motivación para abusar. La carencia de herramientas propias para enfrentar las necesidades y las demandas de los niños -en sus diferentes etapas de desarrollo- para enfrentar los retos de la relación entre generaciones en la época actual y sobrevivit ante el estrés que les impone el ambiente, son factores de riesgo en la motivación para abusar. La segunda precondición es el bloqueo de las inhibiciones del maltratante en potencia. lo que puede suceder como producto de emociones intensas, consumo de alcohol o drogas y lo dispongan a pasar del deseo a la acción. Para avanzar en el proceso y concretar su acción, el sujeto motivado y desinhibido debe contar, además, con la tercera precondición, que es el bloqueo de los inhibidores externos: en otras palabras, un abusador potencial motivado, inserto en una comunidad, familiar o social no preparada para intervenir inhibiendo el proceso abusivo, estará en la mejor posición para abusar. De acuerdo al modelo propuesto, el maltratador potencial, motivado, desinhibido e inserto en una comunidad que no esté en condiciones de actuar como inhibidor externo, necesita tener acceso, estar en una relación de poder con un niño, niña o adolescente vulnerables. La vulnerabilidad de ciertas personas menores de edad constituye la cuarta precondicion en el proceso abusivo. La propuesta contempla que no todas las personas menores de edad son igualmente vulnerables; la falta de información, el aislamiento, la situación social desventajosa, son factores de riesgo que frecuentemente contribuyen a tal vulnerabili$\operatorname{dad}^{13.16}$

\section{Efectos de la violencia sobre los niños}

Los efectos de la violencia familiar sobre el niño se pueden expresar en distintos ámbitos, a corto plazo (físicos, sobre el desarrollo y psi- 
quiátricos) o a largo plazo (transmisión intergeneracional de la violencia, aumento de la criminalidad, desórdenes psiquiátricos y otras complicaciones psicosociales). Debido a que la violencia entre los padres y el abuso infantil con frecuencia coexisten, las consecuencias suelen ser acumulativas para el niño al ser a la vez observador y víctima ${ }^{2}$. 14, 22.24.

Las consecuencias del abuso son diversas. En el plano psicosocial se han descrito, entre otras, cogniciones sociales alteradas, baja autoestima, falta de empatía y depresión. También son frecuentes los síntomas de estrés postraumático, agresividad, problemas de conducta, dificultades de aprendizaje, pesadillas con contenido de violencia y aumento de la angustia cuando el niño recuerda algún episodio abusivo. Estos niños pueden ser inhibidos en el plano emocional, y su conducta puede ser regresiva. Otros síntomas son los trastornos del sueño e irritabilidad ${ }^{1,2,8,11-16,20,24-27}$.

En una investigacion prospectiva para evaluar las consecuencias a largo plazo del abuso, se observó que el abuso físico y el abandono o negligencia en la niñez se asociaban en forma significativa con conducta criminal violenta posterior. Sin embargo, no todo niño abusado o abandonado llega a ser un delincuente, criminal o violento. De esto se deduce que la ligazón entre la victimización infantil y la conducta violenta posterior no es universal y a su vez que la transmisión intergeneracional de la violencia no es inevitable ${ }^{2,25}$. Otras consecuencias a largo plazo son el abuso en el consumo de alcohol y drogas ${ }^{8}$, trastornos de personalidad $3,24,26$, desórdenes afectivos ${ }^{1,2}{ }^{2} 24$, etc. Sin embargo, es importante recordar que no existe un tipo de conducta o psicopatología específica que caracterize al sujeto que experimentó maltrato. En las reacciones de los sujetos maltratados frente al abuso pueden contribuir factores tales como el apoyo de otros miembros de la familia, el nivel de inteligencia y la herencia ${ }^{2}$. También la naturaleza del maltrato, el sexo y la edad del niño $14,15,24$.

\section{Repercusiones en niños y adolescentes testigos de violencia}

S6lo recientemente se ha considerado con riesgo de psicopatología a los niños testigos de violencia entre sus padres ${ }^{2,14-16,27,28}$.
Ellos están expuestos a diversas amenazas, entre las que destacan ser agredidos, abandono o suicidio del padre o la madre ${ }^{15}$. Los conflictos y la violencia doméstica surgen, a menudo, en relación al funcionamiento de la mujer como madre o a desacuerdos entre los padres respecto a la crianza de los hijos, la disciplina y las responsabilidades de cada uno en el cuidado de los niños ${ }^{15}$. Si la madre vive con temor hacia su pareja, esto puede deteriorar su capacidad de manejar y responder a las demandas de su hijo, así, las necesidades básicas de apego de un lactante y sus rutinas de alimentación y sueño pueden estar alteradas. El lactante reconocerá su distancia mostrando retraimiento ${ }^{15}$. Los niños preescolares tienden a culparse cada vez que la madre está enojada, se consideran a sí mismos como culpables de la violencia y en muchos casos responsables de la seguridad de su madre. En éstos se ha descrito irritabilidad, temor, inquietud, síntomas somáticos y conducta regresiva. Muchos viven con miedo y ansiedad porque esperan el próximo episodio violento ${ }^{2,15,16 . ~ L o s ~}$ niños en edad escolar pueden ser ambivalentes. por un lado pretenden esconder lo que sucede dentro de su hogar, mientras por otro desean que alguien lo descubra y pueda rescatarlos. A su vez, experimentan culpa porque creen que habrían podido evitar la violencia. Esta experiencia perturba el desarrollo de su autoestima y confianza en el futuro, en una etapa del desarrollo en que tales logros son fundamentales ${ }^{6,15}$.

La adolescencia, que es una etapa en la que los jovenes establecen por primera vez relaciones intimas fuera de sus familias, y que comienzan a practicar su el sexual y los patrones de comunicación aprendidos, puede también marcar el comienzo de la violencia en las relaciones íntimas. Algunos adolescentes, especialmente varones, pueden irse del hogar o involucrarse en conductas delictuales. Algunas jovenes pueden responsabilizarse de la crianza de sus hermanos menores y de las tareas del hogar, con objeto de mantener la tranquilidad y entregar seguridad a su familia. Ellas suclen sentir que deben proteger a su madre y hermanos y que no pueden abandonar el hogar. Por otra parte, el sufrimiento de la madre puede pasar a ser parte de la rutina diaria, los adolescentes pueden volverse indiferentes a ésta, culparla de los problemas familiares $y$ agredirla ${ }^{15}$. Hay numerosos indicios que sugieren que los niños que presencian 
o experimentan violencia muestran una tendencia a ser abusivos o víctimas de violencia en la edad adulta $15,16,20,27$.

\section{Resumen}

La violencia en el interior de la familia es frecuente en nuestra sociedad. Los niños suelen ser testigos y víctimas de diferentes tipos de maltrato (físico, emocional y sexual), que le afectan directa e indirectamente y cuyos efectos pueden estar presentes a lo largo de toda la vida o incluso ser transferidos a generaciones posteriores. Un conocimiento mas profundo de ella puede ayudar a los profesionales a contribuir en mejorar la calidad de vida de estos niños y sus familias.

(Palabras clave: abuso, castigo físico, maltrato, violencia familiar, niñez.)

\section{Referencias}

1. Novoa $F$, De Ia Barra $F$, Alvarez $P$, Gilchrist A, Alvarez N, Gamboa R: Maltrato infantil en Chile. Rev Chil Pediatr 1992; 63 (Supl. 2): 1-12.

2. Kashani J, Daniel A, Dandoy A, Holcomb W: Family violence: impact on children. J Am Acad Child Adolesc Psychiatry 1992; 31: 181-189.

3. Gilchrist A: Aspectos clínicos del $\mathrm{maltrato}$ infantil. Cuadernos Médico Sociales (Chile) $1991 ; 32: 21-27$

4. Siromak A, Krugman R: Physical abuse of children: an update. Pediatr Rev 1994; 15: 394-399.

5. Johnson $C$ : Child abuse and the child psychiatrist. En Garfinkel B, Car]son G, Weller E. Eds. Psychiatric disorders in children and adolescents. WB Saunders Co. Philadelphia 1990: 339-358.

6. Kempe, $R$, Kempe $H$ : Ninos maltratados. Ediciones Morata, Madrid, 1979.

7. Vargas $N$, Lopez $D$. Pérez $P$. Toro G. Zúñga P. Ciocca $P$ : El castigo físico a los nifios: opinión y conducta de los adultos. Rev Med Chil $1993 ; 121 \div 567.573$.

8. Van Hasselt V, Ammerman R, Glancy L. Bukstein O: Maltreatment in psychiztrically bospitalized dually diagnosed adolescent substance abuser. I Am Acad Child Adolesc Psychiatry 1992; 31 : 868-874.

9. De la Barro F, Alvarez P. Toledo V. Rodríguez J, Caris $L$ : Pesquisa de maltrato infantil $y$ juvenil. Rev Chil Pediatr 1995; 66: 112-117.

10. Finkelhor D, Dziuba-Leatherman $J$ : Children as victims of violence: a national survey. Pediatrics 1994: 94: 413-420.
11. Seman. Documento de trabajo $\mathrm{N}^{\circ} 33$. Módulo de sensibilización en violencia intrafaniliar. Santiago, Chile, 1995.

12. Montt $M$, Ulloa $F$, Rojas $G$ : Violencia familiar desde una perspectiva sistémica. Psiquiatrfa 1993: 10: 57. 61.

13. Organización Panamericana de la Salud. Jornada técvica sobre violencia y salud. Relatoría de la mesa redonda sobre violencia contra la niñez y la adolescencia, Washington DC. Doc PAHO/HMP 1994.

14. Fanularo $R$, Fenton $T$, Kinscherff $R$; Child maltreatment and the development of posttraumatic stress disorder. AJDC 1993; 147; 755-760.

15. Wolfe $D$, Korsch $B$ : Witnessing domestic violence during childhood and adolescence: implication for pediatric practice. Pediatrics 1994; 94: 594-599.

16. Dubowitz $H$, King $H$ : Family violence. A child-centered, family-focused approach. Pediatr Clin North Am $1995 ; 42: 153-163$.

17. Minsal. Unidad de Salud Mental. Maltrato infantil. 1995.

18. Barudy $J$; La violencia como principio organizador de la subjetividad individual, familiar y social. Enfoque sistémico del maltrato a níöos. Contribución a las terceras jomadas chilenas de terapia familiar. Santiago. Chile, 1991

19. Alvarez $P$, Olivari $C$ : Terapia coactiva del maltrato infantil: Ia rehabilitación familiar en el conexto judjcial. Psyche 1993; 2: 24-29

20. Wissow $L$. Roter $D$ : Toward effective discussion of discipline and corporal punishment during primary care visits: fondings from studies of doctor-patient interaction. Pediatrics 1994; 94: 587-593.

21. Flynn $C$ : Relationship violence: a model for family professionals. Fam Relations 1987: 36: 295-299.

22. Bermard $M$, Bernard $J$ : Violent intimacy: the family as a model for love relationships. Fam Relations 1983: 32: 283-286.

23. Leventhal J. Garber $R$, Brady $C$ : Identification during postpartum periods of infants who are at risk of child maltreatment. J Pediatr 1989; 194: 481.487

24. Famularo R. Kinscherff R, Fenton T: Psychiatric diagnoses of maltreated children: preliminary findings. I Am Acad Child Adolesc Psychiatry 1992; 31: 863-867.

25. Mrazek P: Child maltreatment. Cur Opin Pediatr 1990; 2: 715-718.

26. Famularo R, Kinscherff R, Fenton $T$, Bolduc S. Child maltreatment histories among runaways and delinquent children. Clin Pediatr 1990: 29: 713-7L8.

27. Heise Lori: Violencia contra la mujer: la carga oculta de salud. Organización Panamericana de la Salud. Washington DC, Nov 1994. OPS/PWD/94-006.

28. Richman $J$, Chapman $M$, Bowen $G$ : Recognizing the impact of marital discord and parental depression on children. A family centered approach. Pediatr Clin North Am 1995; 42: 167-180. 